\title{
Clinical significance of serum levels of microRNA-27a and its correlation with interleukin-10 in patients with multiple organ dysfunction syndrome caused by acute paraquat poisoning
}

\author{
HUIZHEN DAI ${ }^{1}$, HUAN ZHANG ${ }^{2}$, XINXIA ZU $^{3}$, HONGYAN WANG $^{4}$ and LILI WANG ${ }^{5}$ \\ Departments of ${ }^{1}$ Emergency, ${ }^{2}$ Ophthalmology and ${ }^{3}$ Obstetrics, Affiliated Hospital of Weifang Medical University; \\ ${ }^{4}$ Comprehensive Ward, Hetan Hospital in Hanting District of Weifang; ${ }^{5}$ Operation Room, \\ Affiliated Hospital of Weifang Medical University, Weifang, Shandong 261031, P.R. China
}

Received May 24, 2019; Accepted November 6, 2019

DOI: $10.3892 /$ etm.2020.8409

\begin{abstract}
The aims of the present study were to examine the clinical significance of serum microRNA-27a (miR-27a) levels in patients with multiple organ dysfunction syndrome (MODS) caused by acute paraquat poisoning and to investigate the correlation between miR-27a and interleukin(IL)-10. A total of 82 patients with MODS induced by acute paraquat poisoning and 88 healthy controls were recruited in the present study. Reverse transcription-quantitative PCR was used to measure serum miR-27a expression levels in patients with MODS and the control group. IL-10 serum levels were determined using ELISA. Decreased serum miR-27a level and increased IL-10 expression levels were detected in patients with paraquat poisoning compared with healthy controls $(\mathrm{P}<0.001)$. A moderately negative correlation was identified between the serum expression levels of miR-27a and IL-10 ( $\mathrm{r}=-0.5225$; $\mathrm{P}<0.001)$. miR-27a expression level was found to be associated with blood urea nitrogen, partial pressure of carbon dioxide, arterial blood lactic acid, and the acute physiology and chronic health evaluation II score (APACHE II; P<0.05). The area under the curve for miR-27a was 0.946 , with a sensitivity of $86.6 \%$ and specificity of $87.5 \%$ at a cutoff value of 2.10 . The non-survival patient group had lower miR-27a expression levels compared with the survival group $(\mathrm{P}<0.01)$. Multivariate Cox regression analyses suggested miR-27a expression level and APACHE II score were independent prognostic factors for 30 -day mortality $(\mathrm{P}<0.01)$. The present results suggested that serum miR-27a level may be a potential novel diagnostic and prognostic factor for MODS caused by paraquat poisoning. Collectively, miR-27a may be involved in the process of MODS
\end{abstract}

Correspondence to: Dr Huizhen Dai, Department of Emergency, Affiliated Hospital of Weifang Medical University, 2428 Yuhe Road, Weifang, Shandong 261031, P.R. China

E-mail: huizougm255@163.com

Key words: multiple organ dysfunction syndrome, paraquat poisoning, diagnosis, prognosis, microRNA-27a induced by paraquat poisoning by regulating the inflammatory response.

\section{Introduction}

Paraquat is a type of bipyridine compound that is widely used as a herbicide (1). Self-poisoning with pesticides is a major public health problem in developing countries (2). As a result of its widespread use, paraquat is regarded as the main herbicide involved in accidental and intentional poisoning (3). Paraquat poisoning can be fatal after ingestion, and its mortality rate is $80 \%$ after confirmed exposure $(4,5)$. Multiple organ dysfunction syndrome (MODS) is the main cause of mortality induced by paraquat poisoning (6).

MODS occurs in patients with severe infection or trauma, triggering the release of inflammatory mediators (7). The dysregulation of the inflammatory response plays a crucial role in the development of MODS $(8,9)$. Anti-inflammatory treatment is the primary therapy for the early treatment of paraquat poisoning (5). A number of genes that are differentially expressed during the occurrence and development of inflammation are candidate targets for gene therapy, including some microRNAs (miRNAs/miRs) (10). A recent study reported that miR-146a can regulate the occurrence and immune response of lung injury induced by paraquat poisoning, and that interleukin (IL)-6 is involved in the regulation of this process (11).

IL-10 is a pluripotent anti-inflammatory cytokine that plays a crucial role in the immune response (12). He et al (13) reported that IL-10 mRNA expression levels are increased in rats treated with paraquat, suggesting a crucial role of IL-10 in lung injury of poisoned rats. In a previous study investigating sucralfate intervention for paraquat poisoning, IL-10 was shown to be decreased by sucralfate treatment in rats treated with paraquat, and both lung and kidney injuries of the rats were improved (14). The role of miRNAs in regulating IL-10 has been widely studied $(15,16)$. A previous study showed the protective mechanism of miR-98 in hepatocellular carcinoma (HCC) and IL-10 was found to be the target gene of miR-98, therefore miR-98 may suppress the progression of HCC by targeting IL-10 (14). Additionally, miR-27b-3p and miR-340-5p were reported to target the 3'untranslated regions 
(3'UTR) of IL-10 (17). Transfection and transduction assays showed that upregulation of miR-27b-3p and miR-340-5p downregulated IL-10 expression levels (17). Xie et al (18) reported that miR-27a can regulate the inflammatory response of macrophages by targeting IL-10, and the downregulation of miR-27a was considered to be part of a negative-regulatory mechanism to prevent the inflammatory response. In the present study, to investigate the regulatory mechanism of IL-10, the potential miRNA factors regulating IL-10 were predicted using bioinformatics prediction software and databases including miRanda, TargetScan, PiTa, RNAhybrid and PicTar. The present bioinformatic results suggested that miR-27a was a potential regulator for IL-10. The present results suggested that miR-27a may play a role in MODS induced by paraquat poisoning via the regulation of IL-10.

In the present study, the serum expression levels of miR-27a and IL-10 in patients with paraquat poisoning induced MODS were measured and the clinical significance of miR-27a was investigated.

\section{Materials and methods}

Patients and sample collection. A total of 82 patients with MODS induced by acute paraquat poisoning were enrolled and were diagnosed according to the diagnostic criteria reported by Fry (19). Patients were admitted to the Emergency Department of The Affiliated Hospital of Weifang Medical University from June 2011 to December 2016. All patients arrived at the hospital within $24 \mathrm{~h}$ after poisoning; the poison was ingested orally with an estimated volume of $10-80 \mathrm{ml}$. In total, 10-15 ml venous blood was collected from all patients on admission to hospital, and the serum was isolated and stored at $-80^{\circ} \mathrm{C}$ for further analysis. The patients (age, 17-52 years; mean age, $30.52 \pm 6.93 ; 40$ males and 42 females) did not suffer from immune or immune-related diseases such as diabetes, tumors, chronic liver disease, chronic kidney disease and connective tissue disease. All patients were treated based on a standard treatment protocol including gastric lavage, catharsis, hemoperfusion, antioxidants, high-dose intravenous methylprednisolone and cyclophosphamide (20). A further 88 (age, 18-49 years; mean age, 31.31 7.47; 41 males and 47 females) venous blood samples $(5 \mathrm{ml})$ were collected from healthy fasting participants who had healthy examination results at the same hospital, and were used as the control group. The present study was designed under the review and approval of The Ethics Committee of The Affiliated Hospital of Weifang Medical University. Written informed consent was collected from each participant.

Data collection. All data were recorded in a standard collection for each patient within $24 \mathrm{~h}$ of admission, and included: i) Demographic parameters such as age and gender; ii) time interval from paraquat ingestion to admittance in the emergency department; iii) estimated ingestion amount of paraquat; iv) blood biochemical indexes including white blood count (WBC), blood urea nitrogen (BUN), alanine transaminase (ALT) and aspartate transaminase (AST); and v) arterial partial pressure of carbon dioxide $\left(\mathrm{PaCO}_{2}\right)$ and blood lactic acid (Lac), $\mathrm{PaCO}_{2}$ and Lac were measured using i-STAT blood gas analyzer (Abbott Laboratories).
$R N A$ extraction and reverse transcription-quantitative $P C R$ $(R T$-qPCR). Total RNA was extracted from serum samples using TRIzol reagent (Invitrogen; Thermo Fisher Scientific, Inc.) according to the manufacturer's protocol. Reverse transcription was performed using a miScript RT kit (Qiagen $\mathrm{GmbH}$ ), and the conditions were $37^{\circ} \mathrm{C}$ for $15 \mathrm{~min}$ and $98^{\circ} \mathrm{C}$ for 5 min. SYBR green I Master Mix kit (Invitrogen; Thermo Fisher Scientific, Inc.) was used for RT-qPCR to investigate miR-27a expression levels. The following thermocycling conditions were used for the PCR: Initial denaturation at $95^{\circ} \mathrm{C}$ for $5 \mathrm{~min}$; 30 cycles of $95^{\circ} \mathrm{C}$ for $30 \mathrm{sec}, 60^{\circ} \mathrm{C}$ for $30 \mathrm{sec}$ and $72^{\circ} \mathrm{C}$ for $20 \mathrm{sec}$; and a final extension at $72^{\circ} \mathrm{C}$ for $10 \mathrm{~min}$. U6 was used as an internal reference for data normalization of miR-27a expression levels. The relative expression levels of miR-27a were determined in relation to U6 using the $2^{-\Delta \Delta C q}$ method (21). The primers used were as follows: miR-27a forward, 5'-GGGTTCACAGTGGCTA-3' and reverse, 5'-CTC AACTGGTGTCGTGGA-3'; and U6 forward, 5'-CTCGCT TCGGCAGCACA-3' and reverse, 5'-AACGCTTCACGAATT TGCGT-3'.

Bioinformatics analysis. To investigate the regulatory mechanism of IL-10, the potential miRNA factors regulating IL-10 were predicted using bioinformatics prediction software, miRanda (http://www.microrna.org/microrna/home.do; date of access: August 2010; Memorial Sloan-Kettering Cancer Center), TargetScan (www.targetscan.org/vert_71/; version 7.1), PiTa (http://genie.weizmann.ac.il/pubs/mir07/mir07_data. html; version 6; Weizmann Institute of Science), RNAhybrid (http://bibiserv.techfak.uni-bielefeld.de/rnahybrid/; accessed September 2017; Bielefeld BioInformatics Service) and PicTar (http://pictar.mdc-berlin.de/; accessed March, 2007). miRanda was used according to the following parameters: Energy value $\leq-14 \mathrm{kcal} / \mathrm{mol}$, score $\geq 80$ (22). TargetScan, predictions were ranked according to the predictive efficacy of the target, using context++ scores of the binding sites (23). PiTa was implemented using high-stringency parameters of 7-8 mers and setting the energy value $\leq-10 \mathrm{kcal} / \mathrm{mol}$ as a general cut-off value (24). RNAhybrid was performed setting the energy value $\leq-20 \mathrm{kcal} / \mathrm{mol}$ (25). Prediction using PicTar was based on the PicTar score and free energy (26). The bioinformatics results suggested that miR-27a was a potential regulator for IL-10 (Fig. 1).

ELISA for IL-10. Serum expression level of IL-10 was measured by ELISA using IBL IL-10 ELISA kit (cat. no. 30147233; IBL International $\mathrm{GmBH}$ ) according to the manufacturer's instructions. The concentration of IL-10 was calculated based on standard curves provided with the kits and results are presented in $\mathrm{ng} / \mathrm{l}$.

Statistical analysis. Data are presented as the mean \pm SD. The measurement data was analyzed using Student's t test and the categorical data were compared using Chi-squared test. The correlation between IL-10 and miR-27a expression levels was assessed using Spearman's correlation coefficient. A receiver operating characteristic (ROC) curve was used to calculate the specificity and sensitivity of miR-27a expression level, and to assess its feasibility to diagnose MODS induced by paraquat poisoning. Multivariate Cox regression analyses was performed 


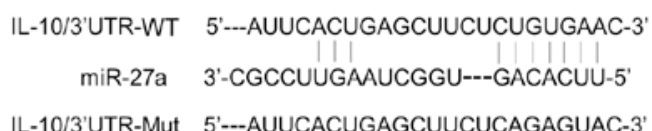

Figure 1. miR-27a is a potential regulator of IL-10. Schematic illustration of the miR-27a targeting site at the 3'UTR of IL-10 gene. miR-27a, microRNA-27a; 3'UTR, 3'untranslated region; IL-10, interleukin-10; WT, wild-type; Mut, mutant.

to examine the association between miR-27a expression level and mortality rate. All data analysis was conducted using GraphPad Prism 5.0 software (GraphPad Software, Inc.) and SPSS version 18.0 software (SPSS, Inc.) $\mathrm{P}<0.05$ was considered to indicate a statistically significant difference.

\section{Results}

Expression level of miR-27a in patients with paraquat poisoning-induced MODS. The serum miR-27a expression levels in all participants were measured using RT-qPCR. The present results suggested that the serum miR-27a expression level was significantly decreased in patients with MODS compared with the healthy controls (Fig. 2; $\mathrm{P}<0.001$ ).

Association of miR-27a expression level with the clinicopathological characteristics of patients with MODS induced by paraquat poisoning. To investigate the clinical role of miR-27a, the correlation between miR-27a expression level and the clinicopathological characteristics of all patients was investigated. All patients were classified into low expression group $(n=47)$ and high expression group $(n=35)$ based on the mean value of serum miR-27a expression level (Table I). The present results suggested that miR-27a expression level was closely associated with BUN ( $\mathrm{P}=0.029), \mathrm{PaCO}_{2}(\mathrm{P}=0.024)$, Lac $(\mathrm{P}=0.012)$ and APACHE II (27) $(\mathrm{P}=0.001)$. However, other clinicopathological parameters showed no significant association with miR-27a expression level including sex, age, time to hospital, estimated amount of paraquat, WBC, BUN, ALT and AST (Table I).

ROC analysis of the diagnostic value of serum miR-27a expression level for MODS induced by paraquat poisoning. A ROC curve is a graphical representation that reflects the correlation between sensitivity and specificity of a laboratory test (28). Based on the serum miR-27a expression levels in patients and controls groups in the present study, a ROC curve was generated to assess the diagnostic value of serum miR-27a expression level for MODS induced by paraquat poisoning. The area under the curve for miR-27a was 0.946 , with a sensitivity of $86.6 \%$ and specificity of $87.5 \%$ at the cutoff value of 2.10 (Fig. 3).

Association of serum miR-27a expression level with mortality rate. All 82 patients with MODS induced by paraquat poisoning were divided into survival group $(n=28)$ and non-survival group $(n=54)$ based on their status after 30 days from admission. The non-survival group had significantly higher levels of WBC, BUN, ALT, AST and Lac, and lower levels of $\mathrm{PaCO}_{2}$ compared with the survival group (Table II; $\mathrm{P}<0.001)$. Additionally, the comparison of traditional score between the survival and non-survival groups showed that

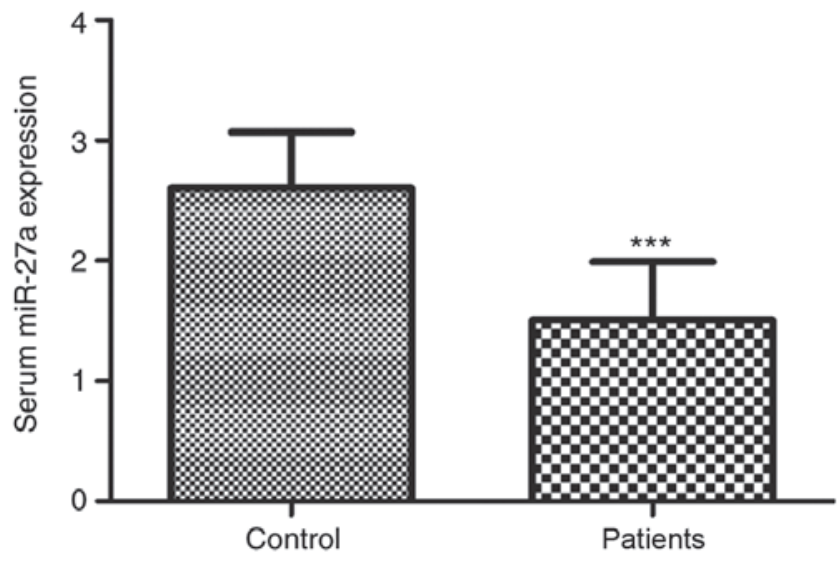

Figure 2. Expression level of miR-27a in patients with paraquat poisoning-induced MODS. Comparison of serum expression level of miR-27a in different groups. ${ }^{* * *} \mathrm{P}<0.001$ vs. the control group. miR-27a, microRNA-27a; MODS, multiple organ dysfunction syndrome.

non-survivors had a significantly higher APACHE II score $(\mathrm{P}<0.001)$. Moreover, miR-27a expression level was significantly different between the survival and non-survival group $(\mathrm{P}<0.01)$, with the non-survival group having more patients with low miR-27a expression levels.

Furthermore, multivariate Cox regression analyses was performed to examine the association between miR-27a expression level and mortality rate. The present results suggested that miR-27a expression level and APACHE II score may be independent prognostic factors to determine overall survival rates (Table III; $\mathrm{P}<0.01$ ).

miR-27a expression level is negatively correlated with IL-10 expression level. The bioinformatic results suggested that miR-27a was a potential regulator for IL-10 (Fig. 1). We further investigated the correlation of serum expression levels of miR-27a with IL-10. ELISA was used for the measurement of the serum IL-10 expression levels. The present results suggested that the serum IL-10 expression level was significantly higher in patients with MODS compared with the healthy controls (Fig. 4A; P<0.001).

The present study also investigated the correlation between serum expression levels of miR-27a and IL-10 in patients with MODS. The present results identified a moderately negative correlation between the serum expressions level of miR-27a and IL-10 (Fig. 4B; r=-0.5225; $\mathrm{P}<0.001$ ).

\section{Discussion}

Paraquat is a widely used herbicide and has a variety of toxic effects (29). The mechanism of acute paraquat poisoning is complex, with many inflammatory factors and cytokines involved (30). Acute paraquat poisoning can lead to a strong inflammatory reaction (31). Previous studies reported that IL-10 mRNA expression level was increased in paraquat poisoned rats, IL-10 level was decreased by sucralfate treatment and the lung and kidney injuries of the rats were also improved $(13,14)$. Therefore, these previous studies demonstrated the important role of IL-10 in paraquat poisoning. IL-10 inhibits the activity of monocyte macrophages, T cells and natural killer cells (32). IL-10 also inhibits the synthesis and release of other inflammatory 
Table I. Association of miR-27a with the clinicopathological characteristics of patients with multiple organ dysfunction syndrome induced by paraquat poisoning.

\begin{tabular}{lcc}
\hline Variable & $\begin{array}{c}\text { Low miR-27a } \\
\text { expression }(\mathrm{n}=47)\end{array}$ & $\begin{array}{c}\text { High miR-27a } \\
\text { expression }(\mathrm{n}=35)\end{array}$ \\
\hline Sex & & 16 \\
Male & 24 & 19 \\
Female & 23 & $31.97 \pm 6.68$ \\
Age, years & $29.45 \pm 6.98$ & $11.11 \pm 5.31$ \\
Time elapsed before admission to hospital, h & $11.04 \pm 4.88$ & $34.77 \pm 19.35$ \\
Estimated volume paraquat ingested, ml & $37.43 \pm 17.81$ & $15.39 \pm 1.19$ \\
WBC & $15.45 \pm 1.55$ & $11.24 \pm 1.28$ \\
BUN & $12.03 \pm 1.80$ & $150.57 \pm 26.26$ \\
ALT & $152.45 \pm 21.08$ & $137.94 \pm 16.97$ \\
AST & $143.83 \pm 21.13$ & 0.950 \\
PaCO & $26.59 \pm 2.64$ & 0.522 \\
Lac & $2.72 \pm 0.54$ & 0.034 \\
APACHE II & $9.98 \pm 2.34$ & $0.99 \pm 2.87$ \\
\end{tabular}

miR-27a, microRNA-27a; WBC, white blood count; BUN, blood urea nitrogen; ALT, alanine transaminase; AST, aspartate transaminase; $\mathrm{PaCO}_{2}$, partial pressure of carbon dioxide; Lac, arterial blood lactic acid; APACHE II, the acute physiology and chronic health evaluation II score.

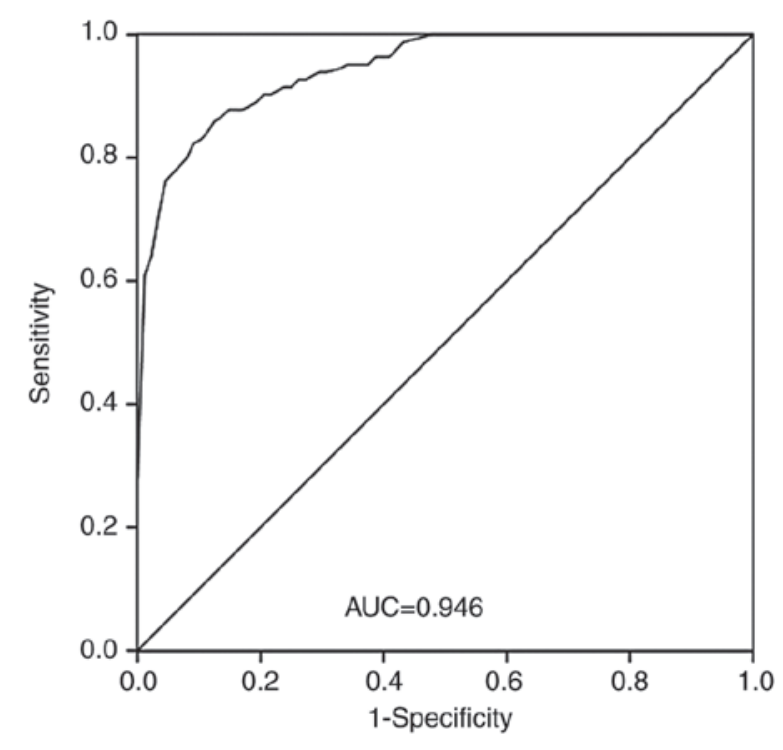

Figure 3. ROC analysis of the diagnostic value of serum miR-27a expression level for multiple organ dysfunction syndrome induced by paraquat poisoning. Diagnostic value of miR-27a using ROC, which had an AUC of 0.946 with a sensitivity of $86.6 \%$ and specificity of $87.5 \%$. ROC, receiver operating characteristic; AUC, area under the curve; miR-27a, microRNA-27a.

cytokines including IL-1 $\beta$, tumor necrosis factor- $\alpha$ (TNF- $\alpha)$ and IL-8 (32). Systemic inflammatory response syndrome (SIRS) occurs when patients have a severe infection or trauma, and compensated anti-inflammatory response syndrome (CARS) may be subsequently triggered (33). The imbalance of SIRS and CARS damages local tissues cells and affects distant organs, eventually leading to MODS (34). A previous study showed that the incidence of MODS in patients with paraquat poisoning was $\sim 84 \%$ (6).
miRNAs are regulatory factors that play crucial roles in regulating growth, maintaining homeostasis of organisms and mediating various pathophysiological processes of diseases, and have become important research topics (35-37). The present bioinformatics results suggested that miR-27a may be a potential regulator for IL-10, thus indicating the potential role of miR-27a in paraquat poisoning. Therefore, a total of 82 patients with MODS induced by paraquat poisoning and 88 healthy controls were enrolled in the present study, and the serum miR-27a expression levels were investigated. The present results suggested the serum miR-27a expression level was significantly lower in patients with paraquat poisoning-induced MODS compared with the healthy controls. The present clinical results suggested that miR-27a may have a significant association with BUN, $\mathrm{PaCO}_{2}$, Lac and APACHE II score. Patients poisoned with paraquat may suffer multiple organ dysfunction of the heart, liver, lungs and kidney (38). BUN is an indicator that reflects kidney function, while $\mathrm{PaCO}_{2}$ and $\mathrm{Lac}$ are important indexes reflecting respiratory function and lung injury (39-41). The present study hypothesized that miR-27a may be involved in kidney and lung injury in patients with MODS induced by paraquat poisoning. Ju et al (42) reported that miR-27a can alleviate acute lung injury in mice by inhibiting inflammation. Additionally, miR-27a was shown to inhibit pulmonary fibrosis in rats (43). Aguado-Fraile et al (44) reported that miR-27a was differentially expressed in acute kidney injury (AKI) patients compared with healthy controls, and serum miR-27a expression level can be used as a biomarker to predict AKI predisposition. Thus, previous studies have shown the important role of miR-27a in kidney and lung injury, in line with the present results.

The present study analyzed the diagnostic and prognostic value of miR-27a in patients with paraquat poisoning-induced MODS. The present results suggested that serum miR-27a expression level was decreased in patients with MODS induced 
Table II. Comparisons between survivors and non-survivors of patients with multiple organ dysfunction syndrome induced by paraquat poisoning.

\begin{tabular}{|c|c|c|c|}
\hline Variable & Survivors $(\mathrm{n}=28)$ & Non-survivors $(\mathrm{n}=54)$ & P-value \\
\hline $\operatorname{miR}-27 \mathrm{a}$ & & & 0.004 \\
\hline Low expression & 10 & 37 & \\
\hline High expression & 18 & 17 & \\
\hline Sex & & & 0.759 \\
\hline Male & 13 & 27 & \\
\hline Female & 15 & 27 & \\
\hline Age, years & $32.00 \pm 7.36$ & $29.76 \pm 6.63$ & 0.166 \\
\hline Time elapsed before admission to hospital, $\mathrm{h}$ & $11.46 \pm 4.73$ & $10.87 \pm 5.21$ & 0.615 \\
\hline Estimated volume paraquat ingested, ml & $34.21 \pm 17.16$ & $37.37 \pm 19.10$ & 0.465 \\
\hline WBC & $14.55 \pm 1.35$ & $15.88 \pm 1.20$ & $<0.001$ \\
\hline BUN & $10.58 \pm 0.89$ & $12.27 \pm 1.64$ & $<0.001$ \\
\hline ALT & $132.93 \pm 24.45$ & $161.35 \pm 15.62$ & $<0.001$ \\
\hline $\mathrm{AST}$ & $129.18 \pm 18.25$ & $147.61 \pm 17.25$ & $<0.001$ \\
\hline $\mathrm{PaCO}_{2}$ & $29.13 \pm 3.03$ & $26.18 \pm 2.09$ & $<0.001$ \\
\hline Lac & $2.01 \pm 0.32$ & $2.89 \pm 0.40$ & $<0.001$ \\
\hline APACHE II & $7.34 \pm 2.20$ & $10.14 \pm 2.00$ & $<0.001$ \\
\hline
\end{tabular}

miR-27a, microRNA-27a; WBC, white blood count; BUN, blood urea nitrogen; ALT, alanine transaminase; AST, aspartate transaminase; $\mathrm{PaCO}_{2}$, partial pressure of carbon dioxide; Lac, arterial blood lactic acid; APACHE II, the acute physiology and chronic health evaluation II score.

Table III. Multivariate Cox regression analysis for miR-27a in patients with multiple organ dysfunction syndrome induced by paraquat poisoning.

\begin{tabular}{lccc}
\hline & \multicolumn{3}{c}{ Multivariate analysis } \\
\cline { 2 - 4 } Variable & HR & $95 \%$ CI & P-value \\
\hline miR-27a & 7.156 & $3.329-15.384$ & $<0.001$ \\
Age & 0.922 & $0.496-1.713$ & 0.797 \\
Sex & 0.620 & $0.320-1.201$ & 0.157 \\
Time elapsed before admission to hospital, h & 1.059 & $0.560-2.004$ & 0.859 \\
Estimated volume paraquat ingested, ml & 1.099 & $0.594-2.034$ & 0.763 \\
WBC & 1.297 & $0.707-2.381$ & 0.401 \\
BUN & 1.292 & $0.670-2.489$ & 0.444 \\
ALT & 1.209 & $0.602-2.429$ & 0.595 \\
AST & 1.385 & $0.713-2.689$ & 0.336 \\
PaCO & 0.624 & $0.199-1.956$ & 0.418 \\
Lac & 1.707 & $0.568-5.125$ & 0.341 \\
APACHE II & 2.730 & $1.282-5.810$ & 0.009 \\
\hline
\end{tabular}

miR-27a, microRNA-27a; WBC, white blood count; BUN, blood urea nitrogen; ALT, alanine transaminase; AST, aspartate transaminase; $\mathrm{PaCO}_{2}$, partial pressure of carbon dioxide; Lac, arterial blood lactic acid; APACHE II, the acute physiology and chronic health evaluation II score; HR, hazard ratio.

by paraquat poisoning. Furthermore, ROC curve analysis suggested that miR-27a may be a sensitive diagnostic biomarker for MODS induced by paraquat poisoning. Previous studies identified that a majority of patients with MODS have lung and kidney injury, and miR-27a was suggested to be involved in organ injury $(43,44)$. Therefore, this may be the possible mechanism for the diagnostic ability of miR-27a in paraquat poisoning. In the present study, according to the overall survival rates after 30 days, patients were divided into survival group and non-survival group. The present results suggested that patients 

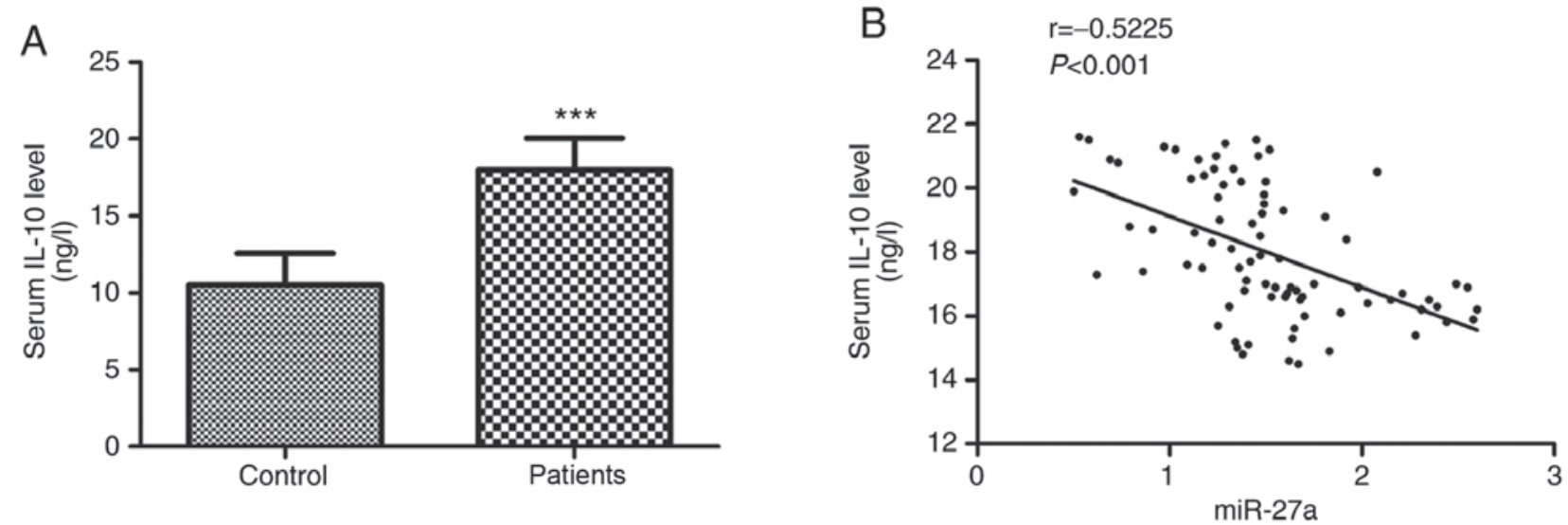

Figure 4. Correlation between serum expression level of miR-27a and IL-10 in patients with MODS induced by paraquat poisoning. (A) Comparison of serum expression level of IL-10 in different groups. (B) Moderate negative correlation between the serum expression levels of miR-27a and IL-10 in patients with MODS induced by paraquat poisoning, $\mathrm{r}=-0.5225 ; \mathrm{P}<0.001 .{ }^{* * *} \mathrm{P}<0.001$ vs. the control group. miR-27a, microRNA-27a; IL-10, interleukin-10; MODS, multiple organ dysfunction syndrome.

in the non-survival group had higher levels of WBC, BUN, ALT, AST and Lac, and a lower level of $\mathrm{PaCO}_{2}$ compared with the survival group; reflecting the reduction in kidney, liver and lung injury. The present results identified that the non-survival group had lower miR-27a expression levels, suggesting that miR-27a expression may be associated with the mortality of patients with paraquat poisoning-induced MODS. Furthermore, the present multivariate Cox regression analysis results suggested that miR-27a expression level may be an independent prognostic factor to predict mortality. As previously reported, APACHE II score can evaluate the prognosis of acute paraquat poisoning (45). The present results suggested that serum miR-27a expression level may have a significant association with APACHE II score, indicating a significant correlation of miR-27a with the severity of paraquat poisoning. Therefore, the present results suggested serum miR-27a may be a useful clinical tool for predicting the outcome of paraquat poisoning.

As indicated by the present bioinformatics results, miR-27a may be a potential regulator of IL-10. The serum expression level of IL-10 was then investigated, and the present results suggested patients with MODS induced by paraquat poisoning had significantly higher IL-10 expression levels compared with healthy controls. In addition, the present results identified a moderate negative correlation between the serum expression levels of miR-27a and IL-10. In paraquat poisoned rats, IL-10 mRNA expression level is upregulated (13). In addition, early gastrointestinal lavage with sucralfate was found to effectively reduce cytokine levels including IL-10, and to improve lung and kidney injury and survival of paraquat poisoned rats (14). Xie et al (18) showed that miR-27a can regulate the inflammatory response by regulating IL-10, and IL-10 was identified to be the target gene of miR-27a in HEK-293 cells. Therefore, results from previous studies are in line with the present results indicating that serum expression level of IL-10 and miR-27a may reflect the inflammatory response and organ damage in MODS induced by paraquat poisoning. The present results suggested that miR-27a may be involved in the process of paraquat poisoning induced MODS by regulating the inflammatory response. Furthermore, a previous study showed that overexpression of miR-27a decreases STAT3 phosphorylation by downregulating IL-10 expression level, and regulates the activation of the JNK1/STAT3 pathway (18). This may be a possible mechanism for miR-27a in the inflammatory response; however, further study is needed to investigate this mechanism.

The present study has several limitations. The clinicopathological parameters of the enrolled patients is not fully comprehensive as a result of incomplete information collection, and other clinicopathological parameters could have been included, such as serum creatinine and serum cardiac troponin I, for a more thorough examination of the association between miR-27a level and different organ dysfunction. In addition, other inflammatory factors involved in the inflammatory response regulated by IL-10 such as IL-6, TNF- $\alpha$, interferon and IL-12 should be examined in patients with MODS. This would be helpful for understanding the inflammatory response in MODS induced by paraquat poisoning. Additionally, the present sample size was relatively small, and further studies with larger sample sizes should be performed.

In conclusion, the present results suggested that increased expression level of IL-10 and decreased expression level of miR-27a in serum may reflect the inflammatory response and organ damage in MODS induced by paraquat poisoning. The serum miR-27a expression level may be a potential novel diagnostic and prognostic factor for patients with MODS induced by paraquat poisoning. In addition, miR-27a may be involved in the process of paraquat poisoning induced MODS by regulating the inflammatory response.

\section{Acknowledgements}

Not applicable.

\section{Funding}

No funding was received.

\section{Availability of data and materials}

The datasets used and/or analyzed during the present study are available from the corresponding author on reasonable request. 


\section{Authors' contributions}

HD made substantial contributions to conception and design, analysis and interpretation of data, draft and revision of the manuscript. HZ and XZ performed the RNA experiments. HW and LW contributed to acquisition of data from patients and samples. All authors were involved in drafting the manuscript and revising it. All authors read and approved the final manuscript.

\section{Ethics approval and consent to participate}

The present study was designed under the review and approval of The Ethics Committee of The Affiliated Hospital of Weifang Medical University (ID of ethics approval: 2011-1-2). Written informed consent was collected from each participant.

\section{Patient consent for publication}

Not applicable.

\section{Competing interests}

The authors declare that they have no competing interests.

\section{References}

1. Flechel A, Jolivet A, Boukhari R, Misslin-Tritsch C, Manca MF, Wiel E, Megarbane B and Pousset F: Paraquat poisoning in Western French Guyana: A public health problem persisting ten years after its withdrawal from the French market. Eur Rev Med Pharmacol Sci 22: 7034-7038, 2018.

2. Eddleston $M$ and Phillips MR: Self poisoning with pesticides. BMJ 328: 42-44, 2004.

3. Zyoud SH: Investigating global trends in paraquat intoxication research from 1962 to 2015 using bibliometric analysis. Am J Ind Med 61: 462-470, 2018.

4. Gil HW, Kang MS, Yang JO, Lee EY and Hong SY: Association between plasma paraquat level and outcome of paraquat poisoning in 375 paraquat poisoning patients. Clin Toxico (Phila) 46: 515-518, 2008

5. Gawarammana IB and Buckley NA: Medical management of paraquat ingestion. Br J Clin Pharmacol 72: 745-757, 2011.

6. Wang W, Li J, Ma G, Li N, Wang P, Xiao Q, Li B, Liu Y, Gao X and Li W: Effect of rhubarb as the main composition of sequential treatment in patients with acute paraquat poisoning: A prospective clinical research. Zhonghua Wei Zhong Bing Ji Jiu Yi Xue 27: 254-258, 2015 (In Chinese).

7. Maier RV: Pathogenesis of multiple organ dysfunction syndrome-endotoxin, inflammatory cells, and their mediators: Cytokines and reactive oxygen species. Surg Infect (Larchmt) 1: 197-205, 2000

8. Kell DB and Pretorius E: To what extent are the terminal stages of sepsis, septic shock, systemic inflammatory response syndrome, and multiple organ dysfunction syndrome actually driven by a prion/amyloid form of fibrin? Semin Thromb Hemost 44: 224-238, 2018

9. Ramirez M: Multiple organ dysfunction syndrome. Curr Probl Pediatr Adolesc Health Care 43: 273-277, 2013.

10. Torrecilla J, Del Pozo-Rodriguez A, Vicente-Pascual M, Solinís MÁ and Rodríguez-Gascón A: Targeting corneal inflammation by gene therapy: Emerging strategies for keratitis. Exp Eye Res 176: 130-140, 2018.

11. Wu W and Li Y: Lung injury caused by paraquat poisoning results in increased interleukin-6 and decreased microRNA-146a levels. Exp Ther Med 16: 406-412, 2018.

12. Tsai TT, Chuang YJ, Lin YS, Wan SW, Chen CL and Lin CF: An emerging role for the anti-inflammatory cytokine interleukin-10 in dengue virus infection. J Biomed Sci 20: 40, 2013.
13. He XY, Sun Q, Li ZW, Lu ZQ, Hong GL, Liang H, Qiu QM and Hu GX: Expression of inflammatory factors in lung tissue of acute paraquat poisoned rats. Zhonghua Lao Dong Wei Sheng Zhi Ye Bing Za Zhi 27: 149-152, 2009 (In Chinese).

14. Junbo Z, Yongtao Y, Hongbo L, Fenshuang Z, Ruyun L and Chun'ai Y: Experimental study of sucralfate intervention for paraquat poisoning in rats. Environ Toxicol Pharmacol 53: 57-63, 2017.

15. Zheng Y, Ge W, Ma Y, Xie G, Wang W, Han L, Bian B, Li L and Shen L: miR-155 regulates IL-10-Producing CD $24{ }^{\text {hi }} \mathrm{CD} 27^{+} \mathrm{B}$ cells and impairs their function in patients with crohn's disease. Front Immunol 8: 914, 2017.

16. Luo XQ, Shao JB, Xie RD, Zeng L, Li XX, Qiu SQ, Geng XR, Yang LT, Li LJ, Liu DB, et al: Micro RNA-19a interferes with IL-10 expression in peripheral dendritic cells of patients with nasal polyposis. Oncotarget 8: 48915-48921, 2017.

17. Rouas R, Merimi M, Najar M, El Zein N, Fayyad-Kazan M, Berehab M, Agha D, Bron D, Burny A, Rachidi W, et al: Human $\mathrm{CD} 8^{+} \mathrm{CD} 25^{+} \mathrm{CD} 127^{\text {low }}$ regulatory $\mathrm{T}$ cells: microRNA signature and impact on TGF- $\beta$ and IL-10 expression. J Cell Physiol 234: 17459-17472, 2019.

18. Xie N, Cui H, Banerjee S, Tan Z, Salomao R, Fu M, Abraham E, Thannickal VJ and Liu G: miR-27a regulates inflammatory response of macrophages by targeting IL-10. J Immunol 193: 327-334, 2014.

19. Fry DE: Multiple system organ failure. Surg Clin North Am 68: 107-122, 1988

20. Feng E, Cheng Y, Tan Z and Wang H: Experience of continuous fluid therapy in successfully rescuing a patient with acute severe paraquat poisoning. Zhonghua Wei Zhong Bing Ji Jiu Yi Xue 31: 1043-1044, 2019 (In Chinese).

21. Livak KJ and Schmittgen TD: Analysis of relative gene expression data using real-time quantitative PCR and the 2(-Delta Delta C(T)) method. Methods 25: 402-408, 2001.

22. Enright AJ, John B, Gaul U, Tuschl T, Sander C and Marks DS: MicroRNA targets in Drosophila. Genome Biol 5: R1, 2003.

23. Agarwal V, Bell GW, Nam JW and Bartel DP: Predicting effective microRNA target sites in mammalian mRNAs. Elife 4, 2015.

24. Kertesz M, Iovino N, Unnerstall U, Gaul U and Segal E: The role of site accessibility in microRNA target recognition. Nat Genet 39: 1278-1284, 2007.

25. Marin RM and Vanicek J: Efficient use of accessibility in microRNA target prediction. Nucleic Acids Res 39: 19-29, 2011.

26. Krek A, Grün D, Poy MN, Wolf R, Rosenberg L, Epstein EJ, MacMenamin P, da Piedade I, Gunsalus KC, Stoffel M and Rajewsky N: Combinatorial microRNA target predictions. Nat Genet 37: 495-500, 2005.

27. Lee H, Lim CW, Hong HP, Ju JW, Jeon YT, Hwang JW and Park HP: Efficacy of the APACHE II score at ICU discharge in predicting post-ICU mortality and ICU readmission in critically ill surgical patients. Anaesth Intensive Care 43: 175-186, 2015.

28. Hoo ZH, Candlish J and Teare D: What is an ROC curve? Emerg Med J 34: 357-359, 2017.

29. Wills BK, Aks S, Maloney GE, Rhee J, Brand R and Sekosan M: The effect of amifostine, a cytoprotective agent, on paraquat toxicity in mice. J Med Toxicol 3: 1-6, 2007.

30. Zhang L, Li Q, Liu W, Liu Z, Shen H and Zhao M: Mesenchymal stem cells alleviate acute lung injury and inflammatory responses induced by paraquat poisoning. Med Sci Monit 25: 2623-2632, 2019.

31. Huang J, Ning $\mathrm{N}$ and Zhang W: Effects of paraquat on IL-6 and TNF- $\alpha$ in macrophages. Exp Ther Med 17: 1783-1789, 2019.

32. Matsui M, Roche L, Soupe-Gilbert ME, Hasan M, Monchy D and Goarant C: High level of IL-10 expression in the blood of animal models possibly relates to resistance against leptospirosis. Cytokine 96: 144-151, 2017.

33. Buerfent BC, Gondorf F, Wohlleber D, Schumak B, Hoerauf A and Hübner MP: Escherichia coli-induced immune paralysis is not exacerbated during chronic filarial infection. Immunology 145: 150-160, 2015.

34. Sauaia A, Moore FA and Moore EE: Postinjury inflammation and organ dysfunction. Crit Care Clin 33: 167-191, 2017.

35. Huang X, Wang L, Liu W and Li F: MicroRNA-497-5p inhibits proliferation and invasion of non-small cell lung cancer by regulating FGF2. Oncol Lett 17: 3425-3431, 2019.

36. Zhang LY, Chen Y, Jia J, Zhu X, He Y and Wu LM: MiR-27a promotes EMT in ovarian cancer through active Wnt/ $\beta$-catenin signalling by targeting FOXO1. Cancer Biomark 24: 31-42, 2019. 
37. Yang ZQ, Wu CA and Cheng YX: Prognostic value of microRNA-133a expression and its clinicopathologic significance in non-small cell lung cancer: A comprehensive study based on meta-analysis and the TCGA database. Oncol Res Treat 41: 762-768, 2018

38. Jiang M, Wang J, Gu S, Cai N, Liu Y, Zhang Q, Xu P and He F: Clinical features and prognosis analysis of the elderly and youth patients with acute severe poisoning. Zhonghua Wei Zhong Bing Ji Jiu Yi Xue 30: 790-794, 2018 (In Chinese).

39. Cakir M, Duzova H, Baysal I, Gül CC, Kuşcu G, Kutluk F, Çakin H, Şeker Ş, İlbeği E, Uslu S, et al: The effect of hypericum perforatum on kidney ischemia/reperfusion damage. Ren Fail 39 385-391, 2017.

40. Shebl E and Burns B: Respiratory Failure: StatPearls, Treasure Island (FL), 2019.

41. B'Chir A, Mebazaa A, Losser MR, Romieu M and Payen D: Intravenous almitrine bismesylate reversibly induces lactic acidosis and hepatic dysfunction in patients with acute lung injury. Anesthesiology 89: 823-830, 1998.
42. Ju M, Liu B, He H, Gu Z, Liu Y, Su Y, Zhu D, Cang J and Luo Z: MicroRNA-27a alleviates LPS-induced acute lung injury in mice via inhibiting inflammation and apoptosis through

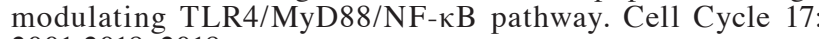
2001-2018, 2018.

43. Liu L, Qian $\mathrm{H}, \mathrm{Hu} \mathrm{K}$, Wang L, Zhang Z, Yin $\mathrm{H}$ and $\mathrm{He} \mathrm{J}$ : miR-27a-3p inhibits pulmonary fibrosis by blocking Wnt3a/ $\beta$-catenin pathway in rats. Xi Bao Yu Fen Zi Mian Yi Xue Za Zhi 34: 1015-1020, 2018 (In Chinese).

44. Aguado-Fraile E, Ramos E, Conde E, Rodríguez M, Martín-Gómez L, Lietor A, Candela Á, Ponte B, Liaño F and García-Bermejo ML: A pilot study identifying a set of microRNAs as precise diagnostic biomarkers of acute kidney injury. PLoS One 10: e0127175, 2015.

45. Huang NC, Hung YM, Lin SL, Wann SR, Hsu CW, Ger LP, Hung SY, Chung HM and Yeh JH: Further evidence of the usefulness of Acute Physiology and Chronic Health Evaluation II scoring system in acute paraquat poisoning. Clin Toxicol (Phila) 44: 99-102, 2006. 